\title{
The representational locus of spatial influence on backward inhibition
}

\author{
Katherine D. ARbuthnotT \\ University of Regina, Regina, Saskatchewan, Canada
}

\begin{abstract}
When one is sequentially switching among three tasks, performance is impaired when tasks alternate (ABA) relative to when one is switching between all three tasks (CBA), an effect known as backward inhibition (BI). $\mathrm{BI}$ is not observed when component tasks are uniquely located in space, however (Arbuthnott, 2005). In this study, the locations of task precues and target stimuli were manipulated independently to determine whether this elimination of $\mathrm{BI}$ is related to distinct cue location or to distinct target location. Results clearly indicated that $\mathrm{BI}$ is eliminated with distinct cue localization independent of the location of target stimuli. This indicates that BI, which reflects suppression of task-set representations, can be influenced by cue characteristics that are associated with task representations.
\end{abstract}

Understanding the mechanisms of cognitive control, such as how individuals direct attention to a single task in a complex environment, is an important goal in cognitive neuroscience. One task that is frequently used to investigate cognitive control is the task-switching paradigm, in which participants perform a number of simple tasks in sequence (see, e.g., Monsell, 2003). One of the control processes that operate in sequential task switching is the suppression of competition from recently performed tasks. The method that was developed by Mayr and Keele (2000) to observe sequential inhibition involves switches among three tasks $(A, B$, and $C)$, and it shows that task repetition on trial $n-2$ in a three-trial sequence (e.g., ABA; alternating-switch) impairs performance relative to nonrepetition on trial $n-2$ in a three-trial sequence (e.g., CBA; two-switch), because of the residual inhibition of task set in the $n-2$ repetition condition. The difference between alternating-switch and twoswitch performance is known as backward inhibition (BI).

The BI effect is robust and has been replicated with several different task characteristics (see, e.g., Altmann, 2007; Arbuthnott, 2005; Gade \& Koch, 2005; Schuch \& Koch, 2003); however, BI is eliminated when one is switching among tasks that are presented at different spatial locations (Arbuthnott, 2005, 2008b; Arbuthnott \& Woodward, 2002). This suggests that there is no inhibition of previous task sets when tasks are presented at unique spatial locations. The effect of spatial location on BI can thus provide an important clue to the operation of sequential inhibition in cognitive control.

The purpose of the present study was to clarify whether the elimination of BI with localized tasks is associated with the localization of target stimuli or the localization of task cues. Arbuthnott (2005) speculated that spatially isolated tasks may be more discriminable from each other, reduc- ing the activation of competing tasks and thus reducing the need for inhibition of competing task sets. Because spatial location was used to cue tasks explicitly in Arbuthnott's (2005) study, the location of cues was emphasized. However, both cues and imperative stimuli for each task were distinct because target stimuli were always presented at the cued location. It is possible that $\mathrm{BI}$ is eliminated by discriminating stimulus-response associations for each task, in which case it would be the spatial location of target stimuli rather than that of cues that underlies this effect. Previous evidence has indicated that BI occurs after the target stimulus has appeared (Mayr \& Kliegl, 2003), rather than during the preparation interval (Hübner, Dreisbach, Haider, \& Kluwe, 2003), lending weight to the proposal that unique target location rather than cue location reduces BI. Furthermore, research using 2:1 mapping of cues to tasks has indicated that switching cues without switching tasks accounts for a considerable portion of switch cost (Logan \& Bundesen, 2004) but does not influence the magnitude of BI (Altmann, 2007; Gade \& Koch, 2008; Mayr \& Kliegl, 2003). There is thus reason to suspect that it is the discriminability of target stimuli rather than of task cues that eliminates BI with spatial cuing.

Evidence also suggests that BI reflects inhibition of global task sets, however, rather than specific stimulusresponse pairings (Hübner et al., 2003; Mayr \& Keele, 2000). Global task set involves the reconfiguration of perception, attention, memory, and motor processes to optimize performance of a given task across all stimuli (Goschke, 2000). Task-set retrieval occurs in response to task cues; thus, it is also possible that the association of specific task sets with location, mediated by spatially distinct presentation of cues, may contribute to the elimination of BI with spatially cued tasks. Furthermore, Druey

K. D. Arbuthnott, katherine.arbuthnott@uregina.ca 
and Hübner (2007) observed BI only when cues and targets were temporally integrated (i.e., cues and targets remained on-screen together), suggesting that sequential inhibition is associated to some degree with cue processing.

In previous demonstrations of this effect, both the task cues and the target stimuli were presented at unique locations for each component task; thus, it was not possible to disambiguate whether target or cue localization was most important to the elimination of BI. The present study investigated this question using independent manipulation of cue and target location: Both the cues and the target stimuli for three tasks were presented either at a common central location or at unique locations for each task, and the locations of cues and target stimuli were not linked. If the elimination of BI by spatial location is due to task-specific stimulusresponse associations for each location, then $\mathrm{BI}$ will be eliminated when target stimuli are presented at unique locations, regardless of the location of the task cues. If cue localization is more central to the elimination of BI, however, suppression will be eliminated by spatially distinct task cues, regardless of the location of target stimuli. Finally, if it is the combination of cue/target location that eliminates BI, the effect will be observed only when both the cues and the targets are presented at unique locations.

\section{METHOD}

\section{Participants}

Fifty-three participants (42 female, 11 male) were recruited either from the research participant pool that is operated by the University of Regina Psychology Department or from the general university community. Participants ranged in age from 18 to 58 years $(M=$ 23.21 years; $S D=8.19$ ), reported normal or corrected-to-normal vision, and spoke English as their first language. Those who were recruited from the research participant pool received course credit in an introductory psychology class, and those who were recruited from the general community received $\$ 10$ in exchange for participation. The session took approximately $60 \mathrm{~min}$ to complete.

\section{Stimuli and Design}

The tasks in this study were three different digit-categorization tasks: parity (odd or even), magnitude (greater or less than 5), and prime-number status (prime or multiple). Stimulus digits included $2,3,4,6,7$, and 9 , which provided equal numbers of stimuli for each bimodal judgment, without complete category overlap for the three tasks. A single digit was displayed on each trial. To indicate which judgment to perform, response-option precues (less/more, odd/even, prime/multiple; visual angles were approximately $4^{\circ} \times 0.7^{\circ}$ ) were displayed $1,000 \mathrm{msec}$ prior to the appearance of the target digit.

As in previous demonstrations of the effect of spatial localization on BI, each task was presented at either a unique location or a common central location. For the common-location conditions, cues were presented slightly above the vertical center of the screen, and the digit stimulus, about $6 \mathrm{~mm}$ by $6 \mathrm{~mm}$ in size, was presented at screen center. For the distinct-location conditions, the cue, the target, or both were presented at one of three locations on the computer screen (center top, bottom left, or bottom right). An example of stimulus layouts for each condition is illustrated in Figure 1. The three locations defined a $10-\mathrm{cm}$ equilateral triangle with its apex at the top of the screen (approximately $11^{\circ}$ of visual angle per side of the virtual triangle). Cues and target stimuli were presented at the center top location for the magnitude task, at the bottom left location for the prime-number-status task, and at the bottom right location for the parity task. At each location, the cues were presented slightly above, and centered over, the location at which the target stimuli would be presented in the distinct-location condition.
Common Cue/Common Target

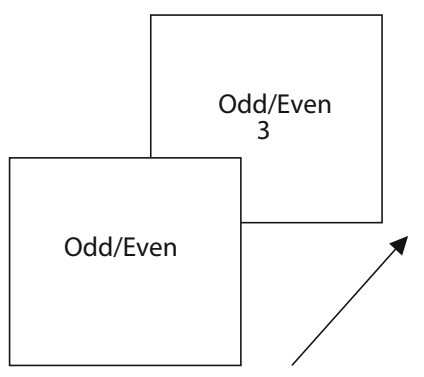

Distinct Cue/Common Target

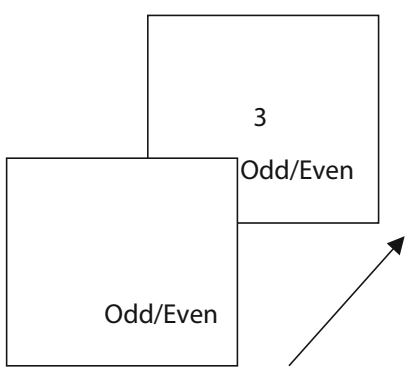

Common Cue/Distinct Target

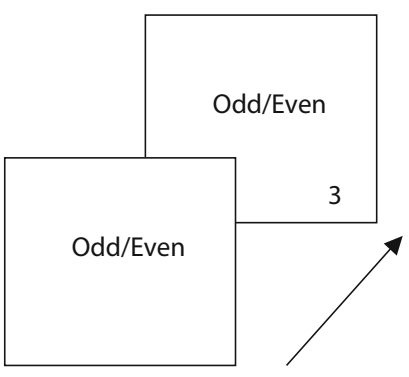

Distinct Cue/Distinct Target

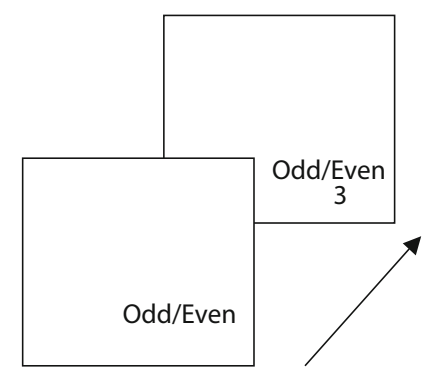

Figure 1. Stimulus layouts. 
For each combination of cue- and task-location condition, two 72trial blocks were presented consecutively. Prior to the first block of each cue/target-location condition, participants received 75 practice trials in that condition. Within each block of trials, each target digit appeared four times for each task. The cue/target-location conditions were presented in a consistent order: common/common; distinct/ distinct; distinct/common; and common/distinct.

The task for each trial was selected randomly, resulting in four possible three-trial sequences: alternating-switch (e.g., BAB), twoswitch (e.g., $\mathrm{CAB}$ ), one-switch (e.g., AAB), and no-switch (e.g., $\mathrm{ABB}$ ). The primary focus in this study was on the alternating-switch and two-switch trials, for the 2 (cue location: common, distinct) $\times$ 2 (target location: common, distinct) $\times 2$ (switch condition: twoswitch, alternating-switch) design. The no-switch and one-switch trials were also analyzed, however, to examine the influence of cue and target location on standard switch cost. All factors were tested within participants.

\section{Apparatus and Procedure}

Participants were tested individually, in a small room, and with the experimenter present. Stimuli were presented on an IBM-compatible computer that was connected to a monitor that displayed black characters against a white background. For each trial, the cue appeared and remained on-screen for $1,000 \mathrm{msec}$, then was joined by the digit stimulus, which remained on-screen until the participant's keypress response (i.e., cue-target interval $=1,000 \mathrm{msec}$ ). $\mathrm{BI}$ is associated with residual switch cost (Arbuthnott \& Frank, 2000; Mayr \& Keele, 2000), so a long cue-target interval was used to isolate this aspect of switch cost. For each trial, both the digit and the task were randomly selected, without replacement, from a set of 72 trials.

Responses were made using all keys of a six-button response box, accurate to $1 \mathrm{msec}$. Univalent response options were used for each task to enable discrimination of wrong-task errors (e.g., judging 3 to be "odd" when a magnitude judgment was cued) and decision errors (e.g., judging 3 to be greater than 5 in a magnitude judgment trial). Responses were made using the same left and right fingers for each task: Magnitude judgment responses were made with the ring fingers (left for less, right for greater), prime judgments were made with the middle fingers (left for prime, right for multiple), and parity judgments were made with the index fingers (left for odd, right for even). The left-right orientation of the responses was chosen to match the order of the response/option cues. Each pair of keys (i.e., task assignment) was marked with a different color (red for magnitude judgment, blue for prime judgment, and yellow for parity judgment).

The cue for a subsequent trial appeared $50 \mathrm{msec}$ after the participant's response on the previous trial (i.e., 50-msec response-cue interval, RCI). A short RCI was also chosen to maximize BI (Gade $\&$ Koch, 2005), enabling more sensitive examination of the effect of cue and target location on BI.

Participants were instructed to respond as quickly and as accurately as possible. Instructions also emphasized that a task cue would appear before the digit appeared to allow participants to prepare for the next task. For trials on which an error was made, the error was recorded, and the trial was re-presented immediately to prevent loss of trials following an error. The response time (RT) for the second presentation of the error trial was not collected.

To familiarize themselves with the procedure in each condition, participants completed 75 practice trials prior to the first block of each condition. Prior to the first such practice trials, 10 training trials for each judgment task were presented, using the central screen location for both the cues and the targets. The order of single-task practice was magnitude, prime, and parity judgments.

\section{RESULTS}

The first two trials in each block were excluded from analysis. The data from 1 participant were excluded because of a very high error rate (37\%), and the data from 2 additional participants were excluded because they were over 45 years of age. The mean age of the remaining participants ranged from 18 to 42 years, with a mean of 21.94 years $(S D=5.58)$.

Trials were coded for switch condition by first designating the 2 three-trial sequences (i.e., two-switch, CAB; alternatingswitch, BAB). Remaining trials were then designated as noswitch (e.g., ABB) or one-switch trials (e.g., AAB). RTs that were more than $2.5 S D$ from each switch-condition mean were discarded as outliers (approximately $2.6 \%$ of trials). Mean RTs and error rates for each switch condition are presented by cue/target-location condition in Table 1 .

\section{Backward Inhibition}

Correct RTs were analyzed using a 2 (switch condition: two-switch, alternating-switch) $\times 2$ (cue location: common, distinct $) \times 2$ (target location: common, distinct) ANOVA. This analysis indicated main effects of all three factors [cue location, $F(1,49)=53.07, p<.001$; target location, $F(1,49)=19.06, p=.001$; switch condition, $F(1,49)=5.34, p=.025]$. For both cue and target location, RTs were faster for the distinct location (cue, $978 \mathrm{msec}$; target, $1,000 \mathrm{msec}$ ) than for the common location (cue, $1,125 \mathrm{msec}$; target, $1,103 \mathrm{msec}$ ). The switch-condition effect indicated that alternating-switch trials were slower $(1,067 \mathrm{msec})$ than two-switch trials $(1,036 \mathrm{msec})$.

These main effects were qualified by significant twoway interactions of cue location with both switch condition and target location. The interaction of cue and target location $[F(1,49)=22.62, p<.001]$ arose because RTs were slower when both the cue and target appeared at a common location in the center of the screen $(1,229 \mathrm{msec})$ than they were in any other combination $(978,1,020$,

Table 1

Mean Response Times (RTs, in Milliseconds) and Error Rates (\%) by Cue/Target Location and Switch Condition

\begin{tabular}{|c|c|c|c|c|c|c|}
\hline \multirow[b]{2}{*}{ Location (Cue/Target) } & \multicolumn{2}{|c|}{ RT } & \multicolumn{2}{|c|}{$\begin{array}{l}\text { Wrong-Task } \\
\text { Errors }\end{array}$} & \multicolumn{2}{|c|}{$\begin{array}{c}\text { Decision } \\
\text { Errors }\end{array}$} \\
\hline & $M$ & $S E$ & $M$ & $S E$ & $M$ & $S E$ \\
\hline \multicolumn{7}{|c|}{ No Switch } \\
\hline Common/common & 795 & 30 & 3.7 & 0.5 & 7.1 & 0.6 \\
\hline Distinct/distinct & 640 & 24 & 1.9 & 0.5 & 4.9 & 0.5 \\
\hline Distinct/common & 670 & 24 & 1.8 & 0.5 & 4.6 & 0.4 \\
\hline Common/distinct & 695 & 25 & 2.2 & 0.5 & 5.0 & 0.5 \\
\hline \multicolumn{7}{|c|}{ One Switch } \\
\hline Common/common & 1,146 & 31 & 7.0 & 1.1 & 6.5 & 0.6 \\
\hline Distinct/distinct & 963 & 19 & 4.0 & 0.6 & 5.2 & 0.5 \\
\hline Distinct/common & 894 & 18 & 4.0 & 0.4 & 4.8 & 0.5 \\
\hline Common/distinct & 947 & 19 & 4.2 & 0.6 & 5.1 & 0.6 \\
\hline \multicolumn{7}{|c|}{ Two Switches } \\
\hline Common/common & 1,192 & 31 & 8.7 & 0.6 & 6.8 & 0.7 \\
\hline Distinct/distinct & 995 & 20 & 6.5 & 0.7 & 5.7 & 0.6 \\
\hline Distinct/common & 963 & 16 & 5.7 & 0.7 & 5.1 & 0.7 \\
\hline Common/distinct & 995 & 24 & 6.2 & 0.7 & 5.1 & 0.5 \\
\hline \multicolumn{7}{|c|}{ Alternating Switches } \\
\hline Common/common & 1,266 & 34 & 7.2 & 0.7 & 7.4 & 0.7 \\
\hline Distinct/distinct & 962 & 21 & 5.0 & 0.6 & 5.7 & 0.6 \\
\hline Distinct/common & 993 & 17 & 7.7 & 0.8 & 4.9 & 0.5 \\
\hline Common/distinct & 1,046 & 30 & 7.2 & 0.8 & 6.0 & 0.7 \\
\hline
\end{tabular}


Switch Condition $\times$ Cue Location

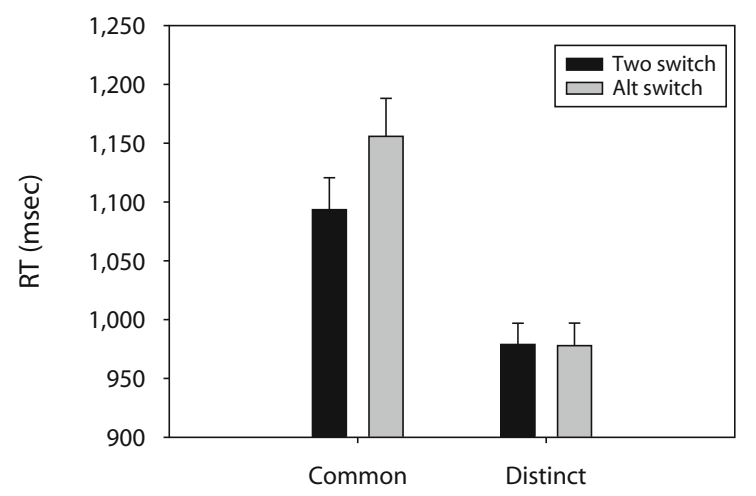

Cue Location

Figure 2. Cue location $\times$ switch condition latency. Error bars $=$ $1 S E$ (within participants; Loftus \& Masson, 1994).

and $978 \mathrm{msec}$, for distinct cue/distinct target, common cue/distinct target, and distinct cue/common target, respectively; Tukey's HSD $=118$ ), perhaps because the common/common condition was encountered first; thus, this interaction may reflect a practice effect.

The interaction of cue location and switch condition $[F(1,49)=6.08, p=.017]$ illustrated in Figure 2 indicated that significant BI was observed when cues were presented in a common location (1,093 vs. 1,156 msec for two-switch vs. alternating-switch, respectively), but not when cues appeared at distinct locations for each task ( 979 vs. $978 \mathrm{msec}$ for two-switch vs. alternating-switch, respectively). BI was significant in both the common/common $[74 \mathrm{msec}$; $t(49)=2.26, p=.028]$ and common/distinct [51 msec; $t(49)=2.12, p=.039]$ conditions, but was insignificant in the distinct/common [31 msec; $t(49)=1.53, p=.133]$ and distinct/distinct [ $-33 \mathrm{msec} ; t(49)=-1.23, p=.224]$ conditions. BI was thus eliminated when cues were presented in distinct locations, regardless of the target location. In contrast, the interaction between switch condition and target location was not significant, nor was the three-way interaction $(F<1)$. These results suggest that it is the distinct location of the task cue, rather than the location of the target stimuli, that eliminates BI.

Wrong-task error rates were also analyzed with a 2 (switch condition) $\times 2$ (cue location) $\times 2$ (target location) ANOVA, and the results indicated a significant three-way interaction $[F(1,49)=10.25, p=.002]$ as well as a main effect of cue location $[F(1,49)=6.02, p=.018]$. Wrongtask errors were less frequent in the distinct-cue condition (6.2\%) than when cues were presented at a common location $(7.3 \%)$. Responses were thus both faster and more accurate with distinct cues, ruling out an overall speedaccuracy trade-off.

The three-way interaction arose because errors were more frequent in the common-cue/common-target twoswitch condition (8.7\%) than in the distinct-cue/commontarget two-switch $(5.7 \%)$ and distinct-cue/distinct-target alternating-switch $(5.0 \%)$ conditions $(\mathrm{HSD}=2.9)$. More

generally, BI (more errors in alternating-switch than in two-switch conditions) was not observed when cues and targets appeared at the same location (either common or distinct), but it was observed when cues and targets appeared at different locations (BI of $0.9 \%$ for common-cue/ distinct-target, and $2.0 \%$ for distinct-cue/common-target conditions). The difference between alternating-switch and two-switch was not statistically significant for any condition, however (largest $t=1.92, p=.061$, for the distinctcue/common-target condition). The significant three-way interaction was thus not clearly related to any systematic effect of the independent variables on wrong-task errors.

The parallel analysis of decision-error rates indicated only a significant effect of cue location $[F(1,49)=5.37$, $p=.025]$. Decision errors were more frequent with common cue location $(6.5 \%)$ than with distinct cue location $(5.3 \%)$. Cue location thus influenced the rates of both types of errors. This influence was not clearly modified by switch condition, however, and these error effects will therefore not be discussed further.

\section{Switch Cost}

To determine how standard switch cost was influenced by cue and target location, correct RTs were analyzed using a 2 (switch condition: no-switch, one-switch) $\times 2$ (cue location) $\times 2$ (target location) ANOVA. All three main effects were significant [switch condition, $F(1,49)=95.19$, $p<.001$; cue location, $F(1,49)=65.75, p<.001$; target location, $F(1,49)=18.01, p<.001]$. No-switch trials were faster $(700 \mathrm{msec})$ than one-switch trials $(987 \mathrm{msec})$, showing the standard switch cost effect. As in the BI analysis, RTs were faster in the distinct-location conditions (cue, $792 \mathrm{msec}$; target, $811 \mathrm{msec}$ ) than in the commonlocation conditions (cue, $896 \mathrm{msec}$; target, $876 \mathrm{msec}$ ).

These main effects were qualified by a significant two-way interaction of cue location and target location $[F(1,49)=19.83, p<.001]$ and a significant three-way interaction $[F(1,49)=13.32, p=.001]$. The interaction of cue and target locations indicated that RTs were slower when both the cue and the target appeared at a common location in the center of the screen $(971 \mathrm{msec})$ than in any other combination $(801,821$, and $782 \mathrm{msec}$, for distinctcue/distinct-target, common-cue/distinct-target, and distinct-cue/common-target, respectively; HSD = 102). As with BI, this may reflect a practice effect.

The three-way interaction arose because switch cost was greater when both the cue and the target appeared in the same location (352 msec for common/common; $323 \mathrm{msec}$ for distinct/distinct) than when they appeared in different locations (252 $\mathrm{msec}$ for common/distinct; $223 \mathrm{msec}$ for distinct/common; HSD = 87). Cue and target location thus influenced switch cost differently than they did BI. BI was observed only when cues appeared in a common location, regardless of target location. In contrast, switch cost was observed for all conditions, but it was greatest when cues and targets shared a spatial location.

Wrong-task error rates were also analyzed with a 2 (switch condition: no-switch, one-switch) $\times 2$ (cue location) $\times 2$ (target location) ANOVA, and the results indicated main effects of all three factors [switch condition, 
$F(1,49)=19.84, p<.001$; cue location, $F(1,49)=8.50$, $p=.005$; target location, $F(1,49)=6.13, p=.017]$. Wrong-task errors were more frequent in the one-switch condition ( $4.8 \%$ vs. $2.4 \%)$, when cues were presented in a common location ( $4.3 \%$ vs. $2.9 \%$ ), and when targets were presented in a common location ( $4.1 \%$ vs. $3.1 \%)$. A significant interaction between cue location and target location was also observed in this analysis $[F(1,49)=4.12, p=$ $.048]$, because error rates were greater in the common/ common condition $(5.4 \%)$ than in any other condition $(3.0 \%, 3.2 \%$, and $2.9 \%$ for distinct/distinct, common/ distinct, and distinct/common conditions, respectively).

The analysis of decision-error rates indicated a significant effect of cue location $[F(1,49)=7.41, p=.009]$, as well as a significant interaction of cue location and target location $[F(1,49)=5.34, p=.025]$. Decision errors were more frequent with common cue location $(5.9 \%)$ than with distinct cue location $(4.9 \%)$. As in previous analyses, the cue location $\times$ target location interaction reflected lower accuracy for the common/common condition (6.8\%) than for all others $(5.1 \%, 5.1 \%$, and $4.7 \%$ for distinct/distinct, common/distinct, and distinct/common conditions, respectively); however, the cue location and target location factors did not interact with switch cost for either type of error.

\section{Practice Effects}

Given that the cue-location/target-location conditions were presented in a consistent order (common/common, distinct/distinct, distinct/common, common/distinct), it is likely that the cue location $\times$ target location interactions indicated practice with the task. To examine practice effects, RTs and error rates were collapsed across switch condition for each pair of blocks, and analyzed them in one-way ANOVAs. These analyses indicated significant practice effects for RTs and for both error types [RT, $F(3,147)=$ $36.82, p<.001$; wrong-task errors, $F(3,147)=4.90, p=$ .003 ; decision errors, $F(3,147)=6.03, p=.001]$. As can be seen in Table 2, Blocks 1-2 were slower (HSD = 64) and more error prone (HSD $=1.7$ and 1.4 for wrong-task errors and decision errors, respectively) than all other conditions, which did not differ from each other. Given the consistent pattern of cue location $\times$ target location interactions in all analyses except the BI error analyses, it appears that practice effects selectively influenced the common-cue/common-target condition.

If these practice effects interacted with switch condition, this would be observed as a significant three-way interaction. This was observed only for wrong-task errors in the BI analysis and for RT in the switch-cost analysis. An examination of Table 2 indicates that these effects were not clearly associated with block order. Latency switch costs, for example, were greater for the distinct/distinct condition (Blocks 3-4) than for the distinct/common condition (Blocks 5-6), despite the absence of practice effects between those conditions. Furthermore, BI effects in RT were even less clearly associated with practice, in that BI was significant in both the common/common condition [Blocks $1-2, t(49)=2.26$, $p=.028]$ and the common/distinct condition [Blocks 7-8, $t(49)=2.12, p=.039$ ], but not in the intervening two conditions, consistent with the absence of a significant threeway interaction in that analysis.

\section{DISCUSSION}

The purpose of the present study was to determine whether distinct location of task cues or distinct location of target stimuli eliminates BI. The results clearly indicate that distinct cue location influences BI magnitude. Regardless of the location of target digits, BI was eliminated when task cues were presented at distinct locations. Conversely, presenting target stimuli at distinct locations for each task did not eliminate BI.

Even with very simple tasks, task switching is complex and involves several different representations and processes. With cued switching, processes include cue encoding, task-set retrieval, target encoding, response retrieval and selection, and response execution. Retrieval and selection processes involve both activation and inhibition. Mental representations that arise from these processes include cue representation and task-set representation, which involves category-stimulus, category-response, and stimulus-response associations.

The finding that it is task-specific cue location that eliminates BI provides further evidence of the task components that are influenced by sequential inhibition. BI reflects inhibition of the $n-2$ task set while one is performing the n-1 task (Arbuthnott, 2005, 2008a; Gade \& Koch, 2005; Hübner et al., 2003). The present results suggest that it is both the process of task-set retrieval and the resulting taskset representation that are influenced by spatial cuing. With distinct cue location, for instance, location-task representations are retrieved, and these representations do not interfere sufficiently with other location-task representations during response retrieval and selection to evoke inhibition (as reflected by BI). This is particularly interesting, because inhibition itself does not arise during the task-preparation

Table 2

Mean Response Times (RTs, in Milliseconds), Error Rates (\%), Switch Cost (SC), and Backward Inhibition (BI) by Block

\begin{tabular}{|c|c|c|c|c|c|c|c|c|c|}
\hline \multirow[b]{2}{*}{ Blocks } & \multirow[b]{2}{*}{ RT } & \multirow[b]{2}{*}{$\mathrm{SC}$} & \multirow[b]{2}{*}{ BI } & \multicolumn{3}{|c|}{ Wrong-Task Errors } & \multicolumn{3}{|c|}{ Decision Errors } \\
\hline & & & & Error & $\mathrm{SC}$ & $\mathrm{BI}$ & Error & $\mathrm{SC}$ & BI \\
\hline $1-2(\mathrm{CC})$ & 1,100 & 352 & 74 & 6.7 & 3.3 & -1.4 & 7.0 & -0.6 & 0.6 \\
\hline $3-4$ (DD) & 890 & 323 & -33 & 4.4 & 2.1 & -1.5 & 5.4 & 0.2 & 0.0 \\
\hline $5-6(\mathrm{DC})$ & 880 & 223 & 31 & 4.8 & 2.2 & 2.0 & 4.9 & 0.1 & -0.2 \\
\hline $7-8(\mathrm{CD})$ & 921 & 252 & 51 & 4.9 & 2.0 & 0.9 & 5.5 & 0.1 & 0.0 \\
\hline
\end{tabular}

Note-CC, common/common; DD, distinct/distinct; DC, distinct/common; CD, common/ distinct. 
interval (Hübner et al., 2003; Schuch \& Koch, 2003), when task set is retrieved. Once task-set representation is retrieved during the preparation interval, however, location information from the target stimulus is apparently not integrated into the task-set representation.

The location-task-set representation then influences the subsequent response retrieval and response selection process. BI has been shown to be sensitive to the magnitude of interference from the previous trial, using both manipulations of delay between trials (Gade \& Koch, 2005) and relative task strength (Arbuthnott, 2008a). BI is thus a flexible process, arising when cross-task interference is greatest. I hypothesize that when task-set representations include discriminating spatial information, cross-task interference is reduced, eliminating the need for sequential inhibition.

Several previous studies, using both standard switchcost and BI paradigms, have shown that the degree of overlap between tasks influences the magnitude of switch cost. Reducing the degree of overlap between task components (stimuli, responses) reduces both BI and standard switch cost. For instance, when tasks are unambiguously associated with different stimuli, switch costs are much smaller (Allport, Styles, \& Hsieh, 1994; Rogers \& Monsell, 1995). Similarly, when responses for each task are univalent, BI can sometimes be eliminated (Gade \& Koch, 2007; but see Arbuthnott, 2008a; Arbuthnott \& Frank, 2000). It is thus possible that spatially distinct task cues are simply another instance of reduced task overlap decreasing the need for executive control when switching between tasks. In the present study, task overlap was significantly reduced in the distinct-cue/distinct-target condition (task-specific responses, cue location, and target location), and BI was totally eliminated in this condition. Standard switch cost in the distinct/distinct condition was equivalent to that observed in the common/common condition, however, suggesting that the reduced overlap did not eliminate switch cost - and thus the need for control — in this condition. The two mixed-location conditions are perhaps most informative about the role of simple task overlap in BI, since the numbers of overlapping components were equal in these conditions. If the magnitude of BI was sensitive to the number of overlapping components, independent of which components overlapped, BI should have been equivalent in these two conditions; however, BI was significant in the common-cue/distinct-target condition but not in the distinct-cue/common-target condition. In a general way, of course, task overlap is the source of interference (and thus $\mathrm{BI}$ ), but the present evidence indicates that not all types of overlap are equal. Specifically, task-specific spatial location appears to decrease interference, even when other sources of overlap, such as multivalent stimuli or responses (Arbuthnott, 2005, Experiment 2) are present.

It should be noted that processing of cues in the distinct locations may differ from that of targets in the distinct locations. For cues, location alone indicated the task, so, even though verbal cues were presented, semantic processing was not necessary in these conditions. For targets, conversely, semantic processing was necessary for both distinct- and common-location conditions. This difference could, hypothetically, account for the different influence of cue versus target location on BI. Given the automaticity of reading processes, the relative transparency of verbal versus spatial cuing, and the 1-sec cue-target interval, however, it is likely that participants read the cues, even in the distinct-cue conditions.

Another possible explanation for the present results is that BI reflects residual suppression of the cue representation itself, rather than task-set suppression. The contention of the compound-cue model of switch cost (e.g., Logan \& Bundesen, 2004) is that, with explicit task cuing, the cuestimulus compound becomes directly associated with a response. Switch cost can thus be attributed to repetition benefit for no-switch trials, rather than to reconfiguration (or inhibition) of task set for switch trials. This hypothesis has been tested with 2:1 cue-task mapping paradigms, which allowed independent observation of cue switches and task switches. When this paradigm has been applied to the threetask situation of BI, however, results have clearly indicated that the magnitude of $\mathrm{BI}$ is not influenced by cue switches between trials $n-2$ and $n$ (i.e., the alternating-switch condition). Robust and equal BI was observed with both cue repetition and cue switches (Altmann, 2007; Gade \& Koch, 2008; Mayr \& Kliegl, 2003), indicating that cues themselves are not the representational locus of BI.

The finding that distinct cue, but not target, location eliminates BI also provides evidence against a simple compound-cue account of task switching (Logan \& Bundesen, 2004). If participants were performing the task using compound cue-stimulus-response associations, then distinct target location would serve as well as distinct cue location to activate a location-digit-response compound, because the location-digit compound would be visually present during target encoding. Since this condition did not influence BI, it appears that response was not based on this compound representation.

Logan and Schneider (2006) also hypothesized that the strength of association between cues and tasks may influence switch processes, with weak cue-task associations (nontransparent cues) necessitating mediated retrieval of a task representation, which could influence task switching (and presumably BI) independent of cue processing. The spatial cues that were used in Arbuthnott (2005) were nontransparent, so the results in that study could be attributed to a mediated retrieval process. The cues that were used in the present study had a strong cue-task association (i.e., transparent cues), however, and the results for the distinct-cue conditions were the same as those for the spatial condition in Arbuthnott (2005); thus, it is unlikely that the present results can be attributed to the strength of cue-task associations.

It appears that these results reflect the interaction of two components of task switching: the influence of cues on task-set representations, and the sensitivity of sequential inhibition (as reflected by $\mathrm{BI}$ ) to interference magnitude. First, task-set representations that are retrieved to guide performance on component tasks are apparently influenced by precues. In particular, when spatial cues are used, task-set representations are associated with unique spatial locations. Once task-set representation is retrieved during the preparation interval, location infor- 
mation from the target stimulus is apparently not integrated into the representation. Location can constrain action-relevant representations (Mayr \& Bryck, 2007), eliminating BI for switches among category-judgment tasks. Considerable evidence indicates the importance of spatial location in human cognition (e.g., Lu \& Proctor, 1995), and it is possible that several task-location representations can be activated simultaneously in working memory (Arbuthnott, 2008b). The second factor is that $\mathrm{BI}$, which reflects sequential inhibition, is strongly influenced by the magnitude of interference from task $n-2$ during response processing on trial $n-1$ (see, e.g., Arbuthnott, 2008a; Gade \& Koch, 2005); thus, if tasklocation representations do not greatly interfere with each other, BI is eliminated. Nonspatial discriminating cue-task associations, such as cue color, do not reduce cross-task interference to the same degree that spatial location does (Arbuthnott, 2008b, Experiment 1), and BI is therefore observed with these manipulations.

As has been argued elsewhere (e.g., Arbuthnott, 2008a; Gade \& Koch, 2008), this indicates that BI reflects a flexible inhibitory mechanism that is evident only when and where sequential competition is greatest. This flexibility is shown in the number of manipulations that have been observed to reduce or eliminate BI in some studies but not in others. These factors include response-set overlap (Gade \& Koch, 2007; but cf. Arbuthnott, 2005; Arbuthnott \& Frank, 2000), temporal cue-target overlap (Druey \& Hübner, 2007; but cf. Grange \& Houghton, in press), and spatial cue-target integration (Druey \& Hübner, 2007, Experiment 1; but cf. Arbuthnott \& Woodward, 2002; Mayr, Diedrichsen, Ivry, \& Keele, 2006). When task sets are uniquely associated with spatial locations, which can apparently be accomplished by presenting task cues at distinct locations, competition between tasks during response selection is reduced, eliminating the need for sequential inhibition (Arbuthnott, 2005). Although BI is eliminated with spatial cues for semantic-judgment tasks, however, it is observed when the component tasks involve spatial judgment (Arbuthnott, 2008b, Experiment 3), further indicating the sensitivity of BI to interference.

\section{AUTHOR NOTE}

This work was supported by the Natural Sciences and Engineering Research Council of Canada (NSERC). I thank Aaron Brown and Amanda Sockett for their help with testing participants. Correspondence concerning this article should be addressed to K. D. Arbuthnott, Campion College, University of Regina, Regina, SK S4S 0A2 Canada (e-mail: katherine .arbuthnott@uregina.ca).

\section{REFERENCES}

Allport, A., Styles, E. A., \& Hsieh, S. (1994). Shifting intentional set: Exploring the dynamic control of tasks. In C. Umiltà \& M. Moscovitch (Eds.), Attention and performance XV: Conscious and nonconscious information processing (pp. 421-452). Cambridge, MA: MIT Press.

Altmann, E. M. (2007). Cue-independent task-specific representations in task switching: Evidence from backward inhibition. Journal of Experimental Psychology: Learning, Memory, \& Cognition, 33, 892-899.

Arbuthnott, K. D. (2005). The influence of cue type on backward inhibition. Journal of Experimental Psychology: Learning, Memory, \& Cognition, 31, 1030-1042.

ArbuthnotT, K. D. (2008a). Asymmetric switch cost and backward inhibition: Carryover activation and inhibition in switching between tasks of unequal difficulty. Canadian Journal of Experimental Psychology, 62, 91-100.

ArbuthnotT, K. [D.] (2008b). The effect of task location and task type on backward inhibition. Memory \& Cognition, 36, 534-543.

Arbuthnott, K. [D.], \& Frank, J. (2000). Executive control in set switching: Residual switch cost and task-set inhibition. Canadian Journal of Experimental Psychology, 54, 33-41.

ArbuthnotT, K. D., \& WoodWard, T. S. (2002). The influence of cuetask association and location on switch cost and alternating-switch cost. Canadian Journal of Experimental Psychology, 56, 18-29.

Druey, M. D., \& HüBnER, R. (2007). The role of temporal cue-target overlap in backward inhibition under task switching. Psychonomic Bulletin \& Review, 14, 749-754.

GADE, M., \& КосH, I. (2005). Linking inhibition to activation in the control of task sequences. Psychonomic Bulletin \& Review, 12, 530-534.

GADE, M., \& KocH, I. (2007). The influence of overlapping response sets on task inhibition. Memory \& Cognition, 35, 603-609.

GADE, M., \& KoCH, I. (2008). Dissociating cue-related and task-related processes in task inhibition: Evidence from using a 2:1 cue-to-task mapping. Canadian Journal of Experimental Psychology, 62, 51-55.

GosCHKE, T. (2000). Intentional reconfiguration and involuntary persistence in task set switching. In S. Monsell \& J. Driver (Eds.), Control of cognitive processes: Attention and performance XVIII (pp. 331-355). Cambridge, MA: MIT Press.

Grange, J. A., \& Houghton, G. (in press). Temporal cue-target overlap is not essential for backward inhibition in task switching. Quarterly Journal of Experimental Psychology.

Hübner, M., Dreisbach, G., Haider, H., \& Kluwe, R. H. (2003). Backward inhibition as a means of sequential task-set control: Evidence for reduction of task competition. Journal of Experimental Psychology: Learning, Memory, \& Cognition, 29, 289-297.

Loftus, G. R., \& Masson, M. E. J. (1994). Using confidence intervals in within-subject designs. Psychonomic Bulletin \& Review, 1, 476-490.

LogAn, G. D., \& Bundesen, C. (2004). Very clever homunculus: Compound stimulus strategies for the explicit task-cuing procedure. Psychonomic Bulletin \& Review, 11, 832-840.

LogAN, G. D., \& SCHNEIDER, D. W. (2006). Interpreting instructional cues in task switching procedures: The role of mediator retrieval. Journal of Experimental Psychology: Learning, Memory, \& Cognition, 32, 347-363.

Lu, C.-H., \& Proctor, R. W. (1995). The influence of irrelevant location information on performance: A review of the Simon and spatial Stroop effects. Psychonomic Bulletin \& Review, 2, 174-207.

MAYR, U., \& BRYCK, R. L. (2007). Outsourcing control to the environment: Effects of stimulus/response locations on task selection. Psychological Research, 71, 107-116.

Mayr, U., Diedrichsen, J., Ivry, R., \& Keele, S. W. (2006). Dissociating task-set selection from task-set inhibition in the prefrontal cortex. Journal of Cognitive Neuroscience, 18, 14-21.

MAYr, U., \& KeELE, S. W. (2000). Changing internal constraints on action: The role of backward inhibition. Journal of Experimental Psychology: General, 129, 4-26.

MAYR, U., \& KLIEgL, R. (2003). Differential effects of cue changes and task changes on task-set selection costs. Journal of Experimental Psychology: Learning, Memory, \& Cognition, 29, 362-372.

Monsell, S. (2003). Task switching. Trends in Cognitive Sciences, 7, 134-140.

Rogers, R. D., \& Monsell, S. (1995). Costs of a predictable switch between simple cognitive tasks. Journal of Experimental Psychology: General, 124, 207-231.

SCHUCH, S., \& Koch, I. (2003). The role of response selection for inhibition of task sets in task shifting. Journal of Experimental Psychology: Human Perception \& Performance, 29, 92-105.

(Manuscript received September 11, 2008; revision accepted for publication January 2, 2009.) 